\title{
The Upgrading of the ICARUS T600 Detector
}

\author{
Gian Luca Raselli*广 \\ INFN Sezione di Pavia \\ E-mail: gianluca.raselliepv.infn.it
}

The ICARUS T600 detector is the largest Liquid Argon Time-Projection-Chamber (LAr-TPC) operated to date. It performed a successful three-year physics run at the underground LNGS laboratories, studying neutrino oscillations with the CNGS neutrino beam from CERN, and searching for atmospheric neutrino interactions in cosmic rays. After an intense refurbishing operation, the entire apparatus has been transferred to FNAL (USA), where it will become the far detector of the Short Baseline Neutrino (SBN) program to investigate the possible presence of sterile neutrino states. The T600 detector underwent a significant overhauling process at CERN, introducing new technological developments while at the same time maintaining the already achieved performance. In this contribution, the main activities of the refurbishing operation are described, highlighting the major changes which will affect the ICARUS T600 physics run in the United States.

EPS-HEP 2017, European Physical Society conference on High Energy Physics 5-12 July 2017

Venice, Italy

* Speaker.

${ }^{\dagger}$ On behalf of the ICARUS collaboration. 


\section{ICARUS T600 detector}

The Liquid Argon (LAr) Time-Projection-Chamber (TPC) technology is well suited for experimental studies of neutrino physics, combining a massive and homogeneous target with excellent tracking and calorimetric capabilities. Originally proposed by C. Rubbia in 1977 [1], this technology has been taken to full maturity with ICARUS T600, which still represents the largest LAr-TPC detector ever built. The detector is made of two identical cryostats each housing two faced TPCs sharing a common cathode [2], filled with a total of $760 \mathrm{t}$ of ultrapure liquid argon corresponding to $476 \mathrm{t}$ of active mass. Each TPC has a maximal drift length of $1.5 \mathrm{~m}$, over which a constant electric field $E=500 \mathrm{~V} / \mathrm{cm}$ is present.

Charged particles interacting in the TPC produce both scintillation light and ionization electrons. The measured drift time, combined with the electron drift velocity $(v \approx 1.6 \mathrm{~mm} / \mu \mathrm{s}$ at $E=500 \mathrm{~V} / \mathrm{cm}$ ) provides the event coordinate in the drift direction; the composition of the three views from the TPC wire planes yields the track projection on the anode plane. This information allows obtaining a full 3D reconstruction of tracks, with a spatial resolution of $\approx 1 \mathrm{~mm}^{3}$.

ICARUS T600 took data from 2010 to 2013 in the Gran Sasso Laboratory (Italy), both with atmospheric neutrinos and with CNGS beam from CERN. During the LNGS run, important LArTPC features were demonstrated [3]: the capability to attain unprecedented levels of liquid argon purity [4], the achievement of high efficient electron/gamma separation and the remarkable particle identification capability by exploiting the measurement of $d E / d x$ versus range [5], the possibility to measure the muon momentum by multiple scattering [6].

\section{The Short Baseline Neutrino (SBN) program at FNAL}

A Short Baseline Neutrino (SBN) program is being developed at Fermi National Accelerator Laboratory (FNAL) [7] to give a definitive answer to the "sterile neutrino puzzle". It foresees the deployment along the Booster Neutrino Beam (BNB) of three detectors, all based on the LAr-TPC technique, namely: SBND (112 $\mathrm{t}$ active volume), MicroBooNE (89 t) and ICARUS T600 (476 t), at a distance of $110 \mathrm{~m}, 470 \mathrm{~m}$ and $600 \mathrm{~m}$ from the target, respectively. Such configuration will allow simultaneous observations of neutrino interactions at different distances, by independently measuring both $v_{e}$ appearance and $v_{\mu}$ disappearance oscillation channels. In case of absence of oscillation, it is expected to have perfectly superimposed neutrino spectra at the different detectors; on the contrary any difference may imply a new physics signal. Sensitivity studies demonstrate the possibility to cover the LSND anomaly parameter region with a $5 \sigma$ confidence level in three years of data taking $\left(\approx 6.6 \times 10^{20}\right.$ pot) by all three LAr-TPC detectors. ICARUS will also collect a large sample of $v_{e}$ CC events from the NUMI off-axis beam at $E \approx 2 \mathrm{GeV}$, which will provide valuable information for the future DUNE project.

\section{The ICARUS T600 upgrading program}

To prepare the detector for this new experimental phase, ICARUS T600 underwent a significant overhauling process in the last two years, introducing new technological developments while at the same time maintaining the already achieved performance. This process took place at CERN 
in the framework of the Neutrino Platform activities (WA104/NP01). The refurbishing included: 1) the preparation of new cold vessels and of a new purely passive insulation system;2) the refurbishing of the inner detectors with TPC cathodes with better planarity; 3) the installation of an improved scintillation light detection system; 4) a new, fast, higher-performance read-out electronics.

\subsection{The new insulation system}

The T600 internal TPCs are placed inside two vacuum tight aluminum containers (cold vessels). Built at CERN premises, they are made by extruded aluminum profiles welded together to form each a vacuum tight double-walled container of parallelepiped shape. The two ends of the containers are bolted to allow for the insertion of the wire chambers frames. A reinforced structure will host the outer thermal insulation (warm vessel) based on passive insulation panels made of plywood, polyurethane foam and other composite materials. Panels are produced in Europe and are being installed directly in the detector housing. A cooling shield with bi-phasic nitrogen will be placed in the space between the warm and the cold vessels for the initial cooling of the detector and to prevent that heat coming through the insulation arrives in the LAr volume. The expected heat loss through the insulation is $\approx 6.6 \mathrm{~kW}$ corresponding to $10 \mathrm{~W} / \mathrm{m}^{2}$.

\subsection{The upgrading of the cathode planarity}

Each module houses two TPCs separated by a common cathode made of punched stainlesssteel sheets with $58 \%$ transparency. Each cathode is set at $-75000 \mathrm{~V}$ to produce a $500 \mathrm{~V} / \mathrm{cm}$ electric drift field. During the LNGS run, the non-perfect planarity of the panels (up to $\approx 25 \mathrm{~mm}$ ) resulted in field distortions of the order of percent affecting the electron-drift velocity for particles interacting close to the cathodes. As a result, a small under-estimation of muon momentum measurement via multiple Coulomb scattering (MCS) was detected at $p>3 \mathrm{GeV} / \mathrm{c}$ [3]. Cathode panels underwent a thermal treatment reducing the non-planarity to within few millimeters. These interventions extend the muon momentum measurement by MCS well above the range required by the next short/long base-line neutrino experiments

\subsection{The new light detection system}

The realization of a new scintillation light detection system is a fundamental feature to reject the expected huge cosmic background due to the T600 operation at shallow depth. A sensitivity below $100 \mathrm{MeV}$ of deposited energy, a time resolution of the order of $\approx 1$ ns and a high granularity are required to effectively identify the events associated to the neutrino beam.

The new T600 light detection system consists of 360 Hamamatsu R5912-MOD PMTs deployed behind the 4 wire chambers, 90 units each, as shown in figure 1. This PMT model is characterized by a 10-dynode stage and an 8 in. bialkali photo-cathode with platinum undercoating. A set of 400 units was tested at CERN to verify the PMTs compliance with the required functioning specifications and 360 units were selected for installation in T600. In particular all the PMTs were tested at room temperature and 60 units were also characterized at cryogenic temperature, in a liquid argon bath to verify any feature variation, such as the PMT gain, dark counts and saturation. Measurements were carried out in different dedicated areas at CERN, where a dark-room and a cryogenic test facility were setup. All the PMTs were rated suitable for detecting the LAr scintillation light and a set of 360 units were selected for installation. 


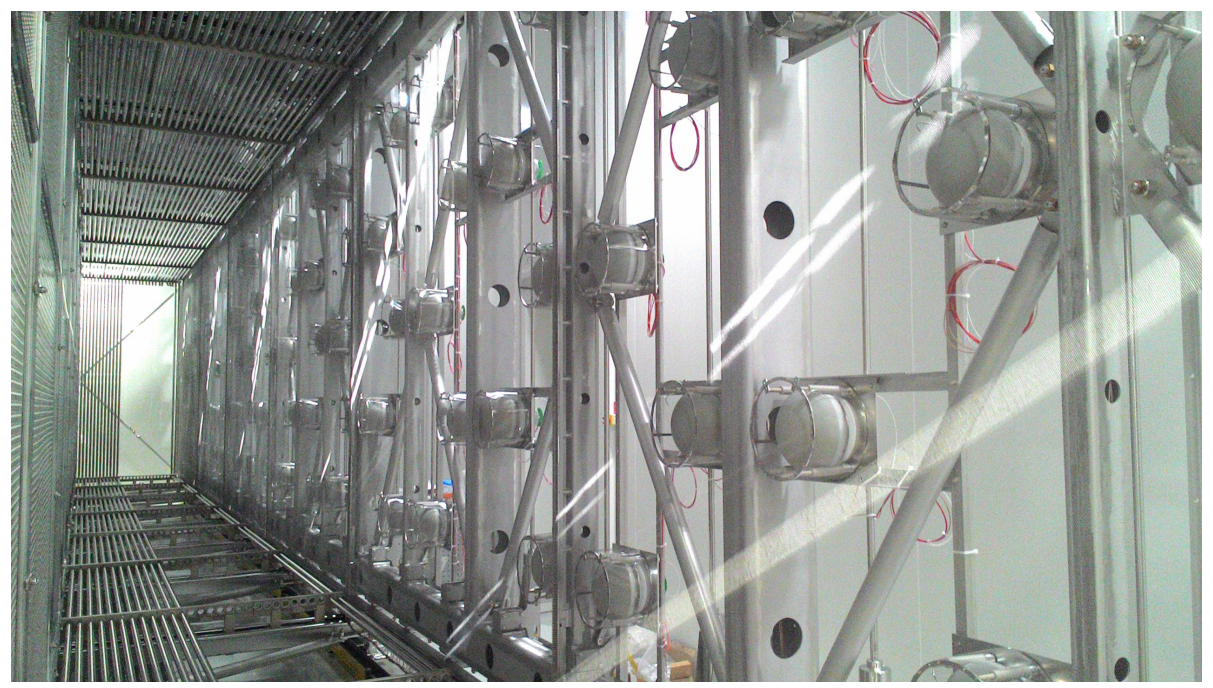

Figure 1: Picture of the PMTs mounted behind one of the four wire chambers.

Since the PMT glass is not transparent to the scintillation light produced in liquid argon $(\lambda=$ $128 \mathrm{~nm}$ ), the units were made sensitive to ultraviolet photons by coating their windows with a proper fluorescent wavelength shifter re-emitting in the visible light frequencies. By means of thermal evaporation, a uniform thickness of $\approx 200 \mathrm{~g} / \mathrm{cm}^{2}$ of Tetra-Phenyl Butadiene (TPB) was deposited on the sensitive surface of each device. For this purpose, a dedicated workshop, hosting a unique evaporation system, was set-up.

A dedicated new design mechanical structure was adopted for the final installation in the TPC. This structure provides the support of each PMT in the correct position and orientation, preventing the electrical interference with the wire planes by means of additional screening grids. Moreover, each support holds up a $50 \mu \mathrm{m}$ optical fiber in front of the PMT sensitive surface for gain and timing equalizazion by means od an external laser. This calibration system, together with the new electronics for the fast sampling of the PMT signals, will allow to obtain a time resolution of events at the nanosecond level.

\subsection{The new wire chamber read-out electronics}

The new wire chamber read-out electronics is based on the adoption of serial 12-bit ADCs, one per channel, in place of the multiplexed ones used in T600 at LNGS [8]. The result consists in a faster read-out system and in a very compact set-up allowing for hosting both analog and digital electronics directly on the feed-through flanges. The main advantage is the synchronous sampling (400 ns) of all channels of the whole detector which is relevent for the $\mu$ momentum measurement by multiple scattering.

A complete read out setup, composed of a flange equipped with 9 TPC digital boards, for a total of 576 channels, was tested at CERN in a 50-liter LAr-TPC. Electronics was equipped with new front-end amplifiers with a sharp signal shaping time $(1.5 \mu \mathrm{s})$ which proved to be effective in the reconstruction of the INDUCTION signals. Offline integration of INDUCTION bipolar signals allows getting unipolar shapes which can be exploited to achieve charge measurement also in the 
INDUCTION view, improving at the same time spatial resolution resulting in clean images both in COLLECTION and INDUCTION views, as shown in figure 2.

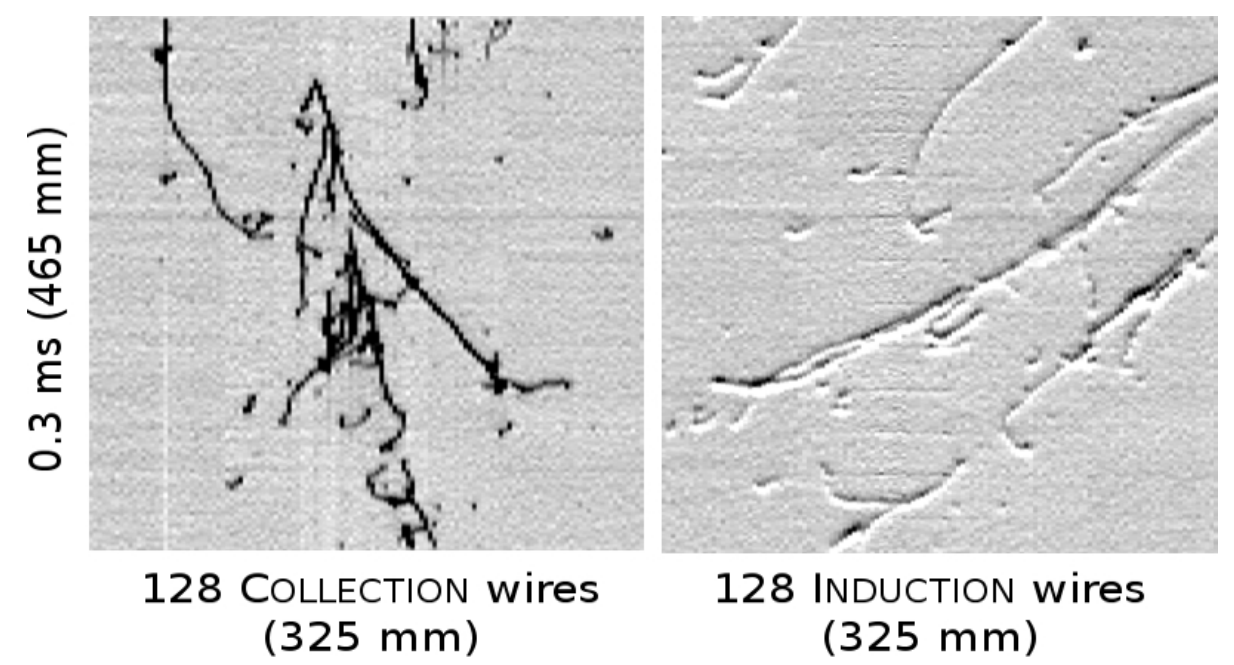

Figure 2: Example of complex shower event recorded at CERN in a 50-liter LAr-TPC equipped with the new electronics. Clean images result both in COLLECTION and INDUCTION views.

\section{Conclusions}

In the last two years the ICARUS T600 detector underwent a significant overhauling process at CERN aimed to prepare the detector for its operation at "shallow depths" at FNAL in the framework of the SBN program. The two T600 internal detectors, completely refurbished, have been installed in new aluminum vessels and have been successfully transported to FNAL. The installation and commissioning will follow, to start as soon as possible the data taking on the Booster Neutrino Beam, to definitively clarify the "sterile neutrino puzzle".

\section{References}

[1] C. Rubbia, The liquid-argon time projection chamber: a new concept for neutrino detectors, CERN-EP-INT-77-8.

[2] S. Amerio et al. Design, construction and tests of the ICARUS T600 detector, Nucl. Instrum. Meth. A 527 (2004) 329-410 [doi:10.1016/j.nima.2004.02.044]

[3] F. Varanini The ICARUS experiment, this conference.

[4] M. Antonello, et al. Experimental observation of an extremely high electron lifetime with the ICARUS-T600 LAr-TPC, (2014) JINST 9 P12006 [doi:10.1088/1748-0221/9/12/P12006]

[5] M.Antonello et al. Experimental search for the "LSND anomaly" with the ICARUS detector in the CNGS neutrino beam, Eur. Phys. J. C (2013) 73:2345 [doi:10.1140/epjc/s10052-013-2345-6]

[6] M.Antonello et al. Muon momentum measurement in ICARUS-T600 LAr-TPC via multiple scattering in few-GeV range, (2017) JINST $12 \mathrm{P} 04010$ [doi:10.1088/1748-0221/12/04/P04010]

[7] FNAL Proposal P-1052 (2014); arXiv:1312.7252 (2013); arXiv:1503.01520 (2015).

[8] Guang Meng The new front end and DAQ of the ICARUS detector, this conference. 